\title{
Cirurgia no câncer colorretal - abordagem cirúrgica de 74 pacientes do SUS portadores de câncer colorretal em programa de pós- graduação lato sensu em coloproctologia
}

\author{
Surgery in colorectal cancer - surgical approach of 74 patients from the Brazilian \\ National Health System with colorectal cancer in a postgraduate program \\ (residency) in coloproctology
}

\begin{abstract}
RODRIGO GUIMARÃES OLIVEIRA ${ }^{1}$, FLAVIA FONTES FARIA ${ }^{1}$, ANTONIO CARLOS BARROS LIMA JUNIOR ${ }^{1}$, FABIO GONTIJO RODRIGUES ${ }^{1}$, MÔNICA MOURTHÉ DE ALVIM ANDRADE ${ }^{2}$, DANIEL MARTINS BARBOSA MEDEIROS GOMES ${ }^{2}$, PETERSON MARTINS NEVES ${ }^{3}$, JOSÉ ROBERTO MONTEIRO CONSTANTINO ${ }^{3}$, ÁUREA CÁSSIA GUALBETO BRAGA³, RENATA MAGALI SILLUZIO FERREIRA ${ }^{3}$, ISABELLA MENDONÇA ALVARENGA $^{3}$, DAVID DE LANNA ${ }^{3}$, RICARDO GUIMARÃES TEIXEIRA ${ }^{3}$, HERALDO NEVES VALLE JUNIOR $^{3}$, SINARA MÔNICA OLIVEIRA LEITE ${ }^{3}$, LUCIANA MARIA PYRAMO COSTA ${ }^{3}$, ILSON GERALDO DA SILVA ${ }^{4}$, GERALDO MAGELA GOMES DA CRUZ ${ }^{5}$
\end{abstract}

${ }^{1}$ Residentes. ${ }^{2}$ Assistentes voluntários. ${ }^{3}$ Assistentes efetivos. ${ }^{4}$ Chefe interino do Serviço de Coloproctologia de Homens da Santa Casa de Belo Horizonte. ${ }^{5}$ Chefe do Serviço de Coloproctologia de Mulheres e do Ambulatório de Coloproctologia da Santa Casa de Belo Horizonte; Coordenador do Grupo de Coloproctologia da Santa Casa de Belo Horizonte e Faculdade de Ciências Médicas de Minas Gerais.

OLIVEIRA RG, FARIA FF, JUNIOR ACBL, RODRIGUES FG, ANDRADE MMDA, GOMES DMBM, NEVES PM, CONSTANTINO JRM, BRAGA ÁCG, FERREIRA RMS, ALVARENGA IM, LANNA DD, TEIXEIRA RG, JUNIOR HNV, LEITE SMO, COSTA LMP, SILVA IGD, CRUZ GMGD. Cirurgia no câncer colorretal - abordagem cirúrgica de 74 pacientes do SUS portadores de câncer colorretal em programa de pós-graduação lato sensu em coloproctologia. Rev bras Coloproct, 2011;31(1): 44-57.

RESUMO: A análise retrospectiva de 74 prontuários de pacientes do Sistema Único de Saúde (SUS), operados de câncer colorretal pelo Residente R2 supervisionado e auxiliado por preceptores, permitiu as seguintes conclusões: a média etária dos pacientes foi 57,2, sendo as sexta e sétima décadas responsáveis por $51,4 \%$ dos pacientes. $O$ câncer retal foi preponderante nas mulheres $(\mathbf{5 4 , 1 \% )}$ ). As localizações mais comuns dos tumores foram no sigmoide $(31,1 \%)$, reto alto $(24,3 \%)$ e ceco $(\mathbf{1 7 , 6 \% )}$. As cirurgias mais realizadas foram a retossigmoidectomia com anastomose colorretal (36,6\%), e hemicolectomia direita com anastomose íleo-transverso (21,7\%). As características anatômicas dos tumores, baseadas na classificação TNM, mais comuns foram: T3 (62,1\%), N0 (59,5\%) e M0 (77,0\%) (p<0,05). O número médio de gânglios encontrados nas peças cirúrgicas foi de 10,4. Foram feitas 63 anastomoses $(85,1 \%)$, das quais 38 (60,3\%) foram mecânicas e 25, manuais (39,7\%). Houve 14 comorbidades (18,9\%), destacando-se a caquexia (oito casos). O índice de complicações cirúrgicas foi de $\mathbf{1 2 , 2 \%}$ (nove casos), sendo as cirurgias que mais causaram complicações as colectomias totais com anastomose íleo-retal $\mathbf{( 4 0 , 0 \% )}$ e as retossigmoidectomias abdominais, com duplo grampeamento $(\mathbf{2 0} \%)$, sendo as complicações mais comuns as fístulas anastomóticas (cinco casos). As complicações (nove) decorreram mais das comorbidades (sete) que do ato cirúrgico (duas). As cirurgias que demandaram menos tempo foram: as laparotomias com ileostomia (média de 75 minutos) e as com colostomia (média de 95 minutos), sendo os maiores tempos ocupados pela proctocolectomia total com ileostomia definitiva (240 minutos) e as hemicolectomias esquerdas com anastomose transverso-retal (240 minutos), sendo o tempo médio equivalente a $\mathbf{1 6 0}$ minutos. As menores peças cirúrgicas foram as decorrentes da cirurgia de Hartmann $(29 \mathrm{~cm})$ e de retossigmoidectomia abdominal $(32 \mathrm{~cm})$; e as mais extensas, as peças de colectomia total com anastomose íleo-retal $(120 \mathrm{~cm})$ e proctocolectomia total com ileostomia definitiva $(150 \mathrm{~cm})$, ficando a média em 34,5 . Houve 12 óbitos $(16,2 \%)$, dois dos quais diretamente relacionados à cirurgia (um caso de deiscência de anastomose e um de evisceração); três relacionados à complicações de ordem clínica (dois casos de TEP e um de broncopneumonia); e sete comorbidades.

Palavras-chave: câncer colorretal; cirurgia colorretal; câncer; SUS.

Trabalho realizado pelo Grupo de Coloproctologia da Santa Casa de Belo Horizonte e Faculdade de Ciências Médicas de Minas Gerais. 


\section{INTRODUÇÃO}

Dentro do Programa de Pós-graduação em Coloproctologia, durante 2009, dois pós-graduandos do segundo ano realizaram, como cirurgiões principais, 129 cirurgias de grande porte, 74 das quais objetivando o câncer colorretal (57,5\%) (Tabela 1 e Figura 1), sempre assistidos, efetivamente, por um ou dois preceptores, conforme o grau de dificuldade da cirurgia. As cirurgias foram realizadas em pacientes do Sistema Único de Saúde (SUS), na Santa Casa de Belo Horizonte, com a presença dos membros do Grupo de Coloproctologia da Santa Casa e Faculdade de Ciências Médicas de Minas Gerais (GCP-SCBH-FCMMG).

\section{OBJETIVO}

Proceder a uma revisão criteriosa de uma casuística de 11 meses (2009), de 74 pacientes operados do câncer colorretal, sendo todos os pacientes do SUS e operados pelo Residente R2, sempre auxiliado por um ou dois preceptores.

\section{MATERIAIS E MÉTODOS}

Os prontuários dos pacientes foram minuciosamente revisados, dando-se absoluta atenção aos seguintes dados: idade; gênero; nome do cirurgião principal (residente R2); auxiliares (preceptores que ajudaram a cirurgia); diagnóstico; resultado da colonoscopia (e biópsia, quando houve); resultado do exame histopatológico das biópsias; preparo intestinal do paciente para a cirurgia (se houve e com qual substância); o tempo de cirurgia; a técnica cirúrgica usada; a forma de anastomose executada (em que lugar anatômico do cólon e/ou reto e com que artefato, se manual ou mecânica); o bisturi e os grampeadores usados na cirurgia; o tipo e a extensão da incisão abdominal; as intercorrências perioperatórias; as dimensões da peça cirúrgica; as complicações pós-operatórias de naturezas cirúrgica e sistêmica; a abordagem das complicações pós-operatórias; o período de internamento hospitalar; o período de permanência no Centro de Terapia Intensiva (CTI); o dia de liberação da dieta; o exame histopatológico da peça cirúrgica; a caracterização dos tumores dentro da classificação TNM (tumor, nodo, metástase); o número

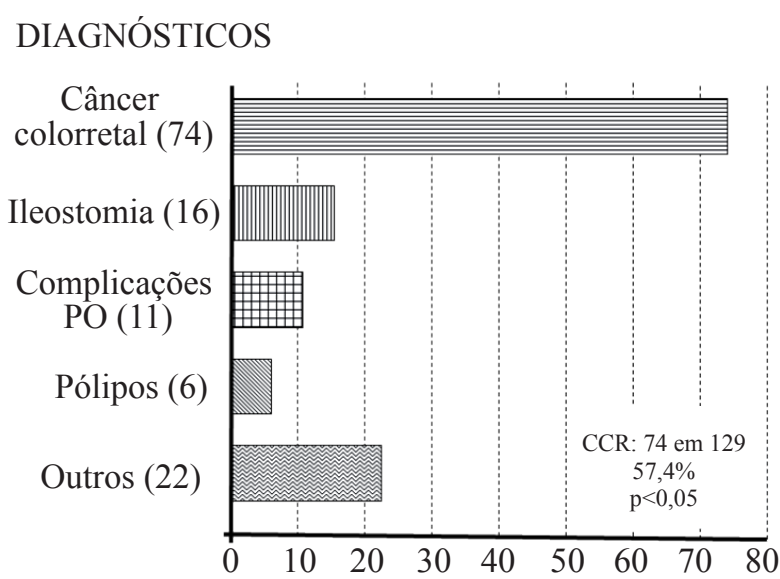

Figura 1. Incidência de pacientes operados de câncer colorretal em um universo de 129 pacientes submetidos a cirurgias ênterocolorretais, por dois médicos residentes em Coloproctologia como cirurgiões principais, no programa de Pós-Graduação "lato sensu"do Grupo de Coloproctologia da SCGH e FCMMG.

Tabela 1. Incidência de pacientes operados de câncer colorretal em um universo de 129 pacientes submetidos a cirurgias êntero-colorretais, por dois médicos residentes em Coloproctologia coko cirurgiões principais, no Programa de Pós-Graduação "lato sensu" do Grupo de Proctologia da SCBH e FCMMG.

\begin{tabular}{|c|c|c|}
\hline $\begin{array}{l}\text { DIAGNÓSTICOS } \\
\text { PRINCIPAIS }\end{array}$ & $\mathbf{N}$ & $\%$ \\
\hline CÂNCER COLORRETAL & 74 & 57,4 \\
\hline ILEOSTOMIA & 16 & 12,4 \\
\hline $\begin{array}{l}\text { COMPLICAÇÕES CIRÚRGICAS } \\
\text { OPERADAS }\end{array}$ & 11 & 8,5 \\
\hline $\begin{array}{l}\text { PÓLJPOS SIGMOIDE (3), CECO } \\
\text { (1), TRANSVERSO (1), RETO(1) }\end{array}$ & 06 & 4,6 \\
\hline $\begin{array}{l}\text { OUTRAS CONDIÇÕES } \\
\text { MÓRBIDAS COLORRETAIS }\end{array}$ & 22 & 17,1 \\
\hline Chron ilean, cólon $\mathrm{c} / \mathrm{Ft}$ e reto $\mathrm{c} / \mathrm{Ft}$ & 4 & \\
\hline Enterite e retite actínica com $\mathrm{Ft}$ & 4 & \\
\hline Hartmann & 3 & \\
\hline Procidência retal & 2 & \\
\hline Megacólon Chacásico & 2 & \\
\hline Endometriose de sigmoide & 2 & \\
\hline $\begin{array}{l}\text { Abdome agudo inflamatório } \\
\text { (apendicite) }\end{array}$ & 1 & \\
\hline $\begin{array}{l}\text { AAP por doença diverticular de } \\
\text { sigmoide }\end{array}$ & 1 & \\
\hline Hidradenite & 1 & \\
\hline Diverticulose colônica difusa & 1 & \\
\hline Fístula de bolsa ileal & 1 & \\
\hline Total & 129 & 100,0 \\
\hline
\end{tabular}


de gânglios encontrados na peça cirúrgica; o número de gânglios comprometidos pela doença; a margem livre de tumor e as comorbidades apresentadas pelos pacientes. Da mesma forma, realizou-se uma correlação entre morbimortalidade e comorbidade, uma vez que os pacientes, em marcante número de vezes, apresentavam estado geral precário, acumulando outras doenças além do tumor colorretal.

\section{RESULTADOS}

\section{Incidência de câncer colorretal e outras doenças colorretais}

Dos 129 pacientes operados por várias doenças colorretais, 74 eram portadores de câncer colorretal $(57,4 \%)$, seguindo outros diagnósticos nosológicos, tais como: ileostomias (16 casos; 12,4\%); complicações de cirurgias anteriores (11 casos; 8,5\%); pólipos colorretais ( 6 casos; $4,6 \%$ ) e outras condições mórbidas colorretais (22 casos; 17,1\%).

\section{Faixa etária e gêneros}

A média etária dos pacientes foi 57,2 anos, com extremos de 27 e 85 anos, sendo as sexta e sétima décadas responsáveis por $51,4 \%$ dos pacientes (Tabela $2 \mathrm{e}$ Figura 2). Houve mais cirurgias em mulheres $(54,1 \%)$ do que em homens $(45,9 \%)$ (Tabela 3 e Figura 3$).$

Tabela 2. Distribuição, por décadas etárias, de 74 pacientes portadores de câncer colorretal submetidos a cirurgias, por dois médicos residentes em Coloproctologia como cirurgiões principais, no Programa de Pós-Graduação "lato sensu”do Grupo de Coloproctologia da SCBH e FCMMG.

\begin{tabular}{llclc}
\hline Idade (anos) & $\mathbf{N}$ & $\mathbf{\%}$ & $\mathbf{\Sigma N}$ & $\mathbf{\Sigma \%}$ \\
\hline$<20$ & 00 & 0 & 00 & 0 \\
$21-30$ & 01 & 1,3 & 01 & 1,3 \\
$31-40$ & 07 & 9,5 & 08 & 10,8 \\
$41-50$ & 15 & 20,3 & 23 & 31,1 \\
$51-60$ & 19 & 25,7 & 42 & 56,8 \\
$61-70$ & 19 & 25,7 & 61 & 82,5 \\
$71-80$ & 10 & 13,5 & 71 & 96,0 \\
$81-90$ & 03 & 4,0 & 74 & 100,0 \\
$91-100$ & 00 & 0 & & \\
Total & 74 & 100,0 & 74 & 100,0 \\
\hline
\end{tabular}

Média: 57,2 anos; Mínima: 27 anos; Máxima: 85 anos; 51-60 anos: 19(25,7); 61-70 anos: 19(25,7); Faixas etárias com $p<0,05$

\section{Localizações dos tumores no intestino grosso}

As localizações dos tumores foram mais comuns no sigmoide ( 23 casos; $31,1 \%$ ), reto alto (18 casos; $24,3 \%$ ) e ceco (13 casos; $17,6 \%$ ), seguidos pelo reto baixo (oito casos; 10,8\%), cólon transverso (três casos; $4,0 \%$ ), cólon ascendente (dois casos; 2,7\%), cólon descendente (dois casos; 2,7\%), recidiva pélvica de câncer retal (quatro casos; $5,4 \%$ ) e carcinomatose com abdome agudo obstrutivo (um caso; $1,4 \%$ ) (Tabela 4 e Figura 4).

\section{Cirurgias realizadas}

As cirurgias realizadas nos 74 pacientes foram as seguintes: retossigmoidectomia com anastomose co-

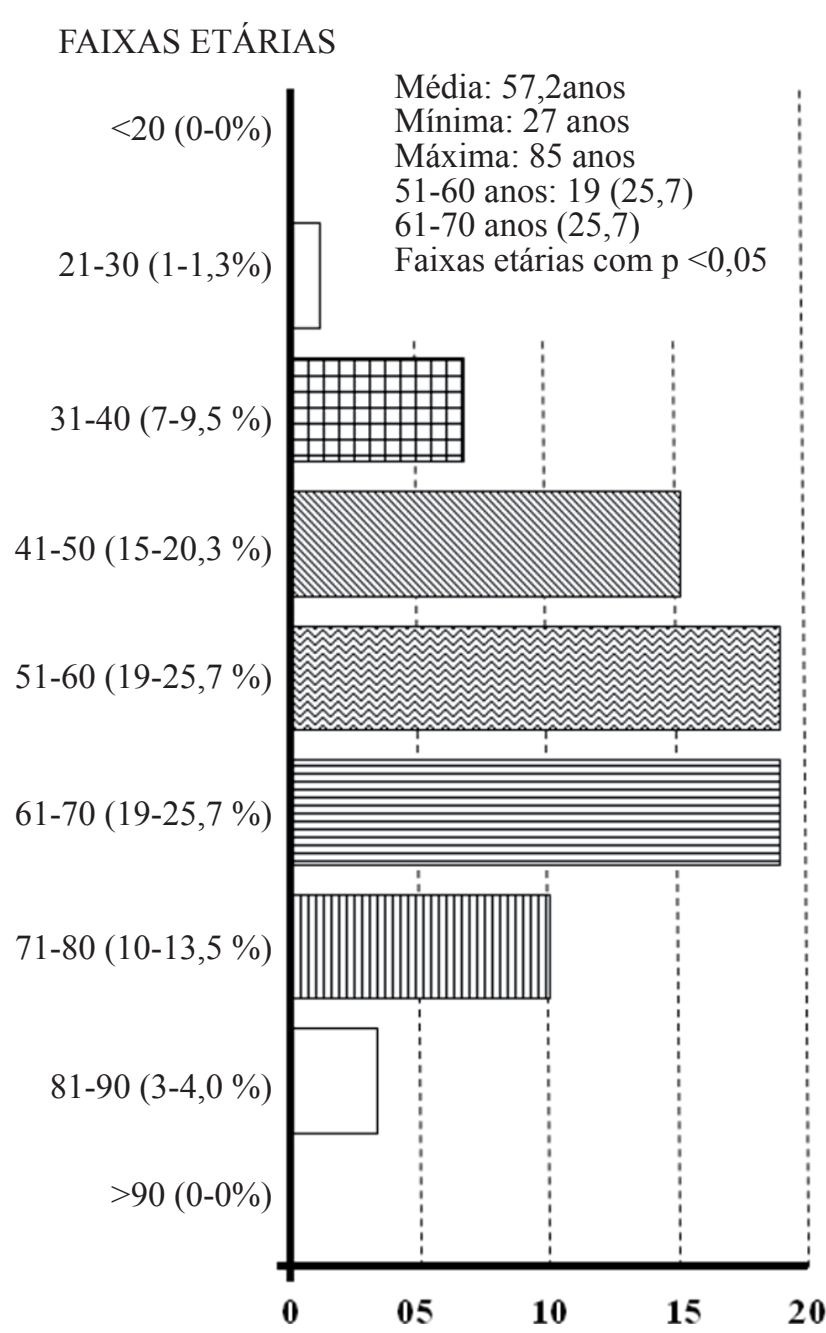

Figura 2. Distribuição, por décadas etárias, de 74 pacientes portadores de câncer colorretal submetidos a cirurgias, por dois médicos residentes em Coloproctologia como cirurgiões principais, no Programa de Pós-Graduação lato sensu do Grupo de Coloproctologia, da Santa Casa de Belo Horizonte. 
Tabela 3. Distribuição, por gênero dos pacientes, de 74 pacientes portadores de câncer colorretal submetidos a cirurgias êntero-clorretais, por dois médicos residentes em Coloproctologia como cirurgiões principais, no Programa de Pós-Graduação "lato sensu"do Grupo de Coloproctologia da SCBH e FCMMG.

\begin{tabular}{lcc}
\hline Gêneros & N & \% \\
\hline Feminino & 40 & 54,1 \\
Masculino & 34 & 45,9 \\
Total & $\mathbf{7 4}$ & $\mathbf{1 0 0 , 0}$ \\
\hline
\end{tabular}

Tabela 4. Distribuição, por localização dos tumores, de 74 pacientes portadores de câncer colorretal submetidos a cirurgias êntero-clorretais, por dois médicos residentes em Coloproctologia como cirurgiões principais, no Programa de Pós-Graduação "lato sensu"do Grupo de Coloproctologia da SCBH e FCMMG.

\begin{tabular}{llc}
\hline CÂCER & $\mathbf{N}$ & $\mathbf{\%}$ \\
COLORRETAL & & 31,1 \\
\hline Sigmoide & 23 & 24,3 \\
Reto Alto & 18 & 17,6 \\
Ceco & 13 & 10,8 \\
Reto baixo & 08 & 4,0 \\
Transverso & 03 & 2,7 \\
Ascendente & 02 & 2,7 \\
Descendente & 02 & 5,4 \\
Recidiva pélvica de & 04 & 1,4 \\
câncer de reto & & \\
Carcinoma com & 01 & 100,0 \\
AAO (enterectomia) & & \\
Total & 74 &
\end{tabular}

CCR: 74 ; sigmoide: $23(31,1 \%)$; reto alto: $18(24,3 \%)$; sigmoide + reto: $41(55,4 \%) ; p<0,05$

lorretal ( 27 casos; 36,6\%); hemicolectomia direita com anastomose íleo-transverso (16 casos; $21,7 \%$ ); colectomia total com anastomose íleo-retal (5 casos; 6,7\%); retossigmoidectomia abdominal com duplo grampeamento (5 casos; 6,7\%); cirurgia de Hartmann (5 casos; 6,7\%); laparotomia e colostomia ( 5 casos; $6,7 \%$ ); laparotomia e ileostomia ( 2 casos; $2,7 \%$ ); hemicolectomia esquerda com anastomose transverso-retal (2 casos; $2,7 \%$ ); proctocolectomia total com ileostomia

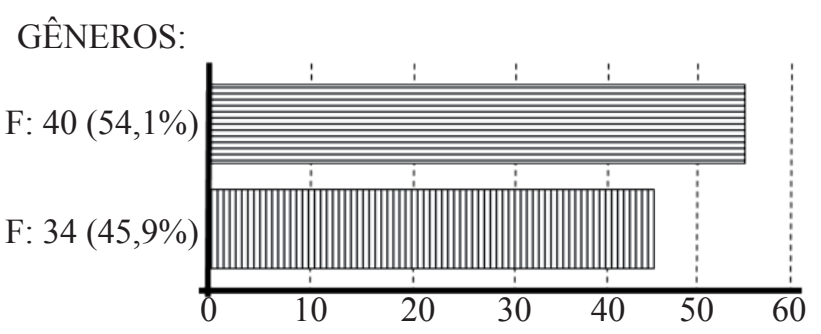

Figura 3. Distribuição, por gênero dos pacientes, de 74 pacientes portadores de câncer colorretal submetidos a cirurgias ênterocolorretais, por dois médicos residentes em Coloproctologia como cirurgiões principais, no Programa de Pós-Graduação, "lato sensu"do Grupo de Coloproctologia da SCBH e FCMMG.

\section{TOPOGRAFIAS DOS TUMORES COLORRETAIS}

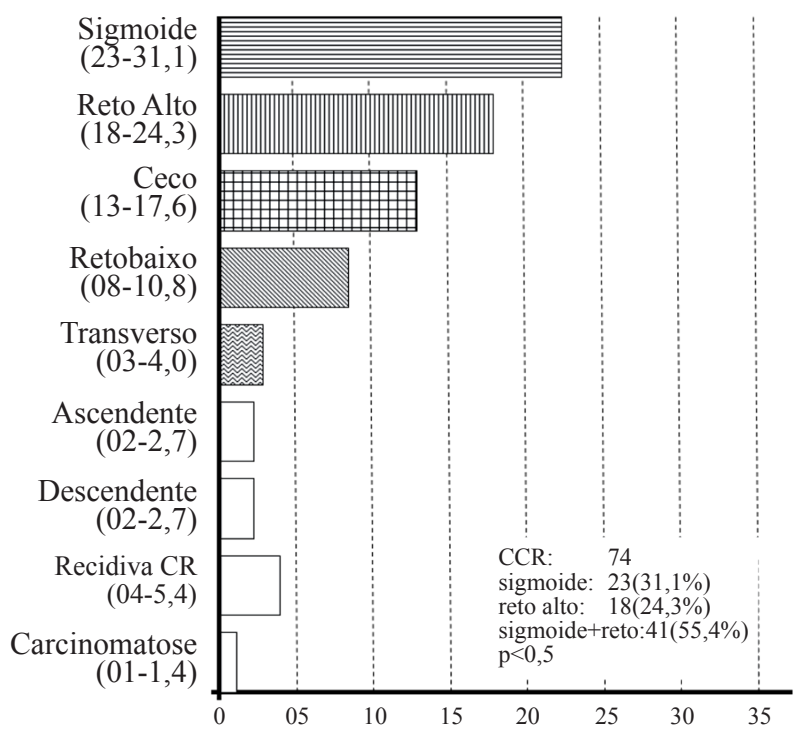

Figura 4. Distribuição, por localizações dos tumores, de 74 pacientes portadores de câncer colorretal, submetidos a cirurgias êntero-colorretais, por dois médicos residentes em Coloproctologia como cirurgiões principais, no Programa de Pós-Graduação lato sensu do Grupo de Coloproctologia da SCBH e FCMMG.

definitiva ( 2 casos; 2,7\%); amputação abdominoperineal com colostomia definitiva ( 2 casos; $2,7 \%$ ); panhisterectomia com enterectomia e ressecção de metástases ( 2 casos; $2,7 \%$ ) e enterectomia com liberação de aderências (1 caso; 1,4\%) (Tabela 5 e Figura 5).

\section{Classificação TNM dos tumores}

As características anatômicas dos tumores, baseadas na classificação TNM, foi a seguinte (Tabela 
CIRURGIAS

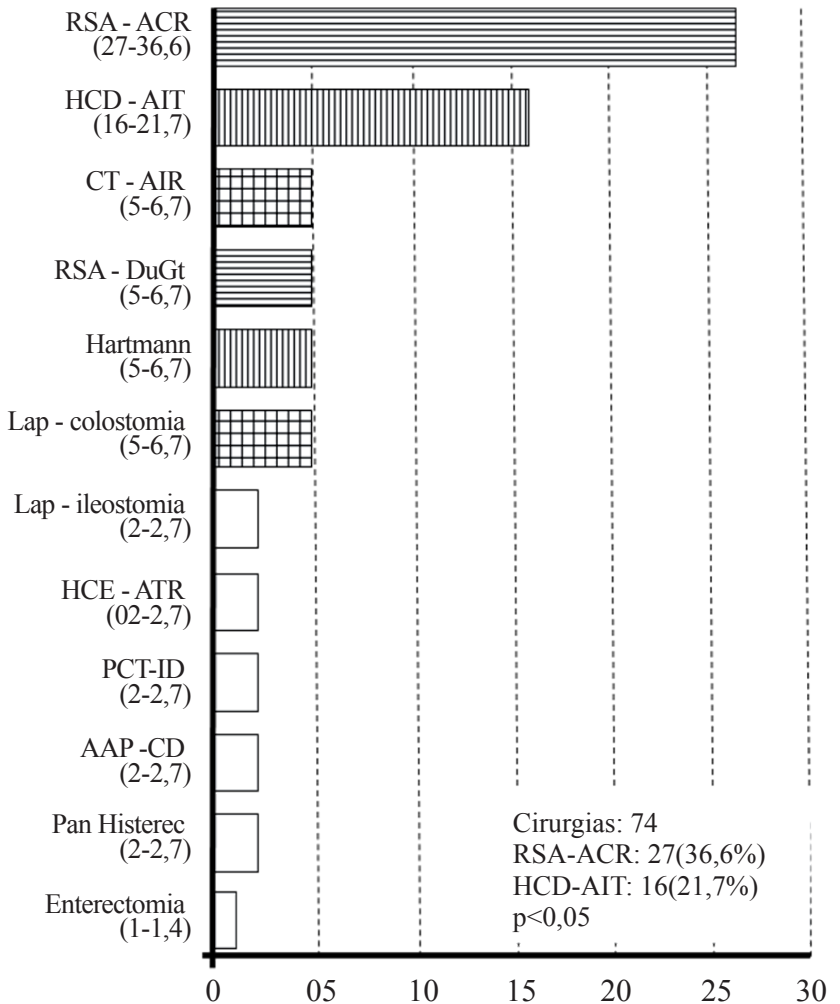

RSA-ACR: retossigmoidectomia abdominal com anastomose colorretal; HCD-AIT: hemicolectomia direita com anastomose ileotransverso; RSA-ACR-DuGr: RSA-ACR com duplo grampeamento; CT-AIR: colectomia total com anastomose íleo-retal; HCE-ATR: hemicolectomia esquerda com anastomose transverso-retal; PCTID: proctocolectomia total com ileostomia definitiva; $A A P-C D$. amputação abdominoperineal com colostomia definitiva.

Figura 5. Distribuição, por cirurgias a que foram submetidos, de 74 pacientes portadores de cancer colorretal, submetidos a cirurgias êntero-colorretais, por dois médicos residentes em Coloproctologia como cirurgiões principais, no Programa de Pós-Graduação lato sensu do Grupo de Coloproctologia da SCBH e FCMMG.

6 e Figura 6): T0 (1 caso; 1,3\%), T1 (1 caso; $1,3 \%)$, T2 (13 casos; 17,7\%), T3 (46 casos; 62,1\%) e T4 (13 casos; 17,6\%); N0 (44 casos; 59,5\%), N1 (23 casos; $31,1 \%$ ) e NX (7 casos; 9,4\%); M0 57 casos; 77,0\%, M1 (13 casos; $17,6 \%)$ e MX ( 4 casos; $5,4 \%)$. Verificase, assim, que 46 tumores $(62,1 \%)$ eram T3 $(\mathrm{p}<0,05)$, $44(59,5 \%)$ não apresentavam metástases ganglionares $(\mathrm{p}<0,05)$ e $57(77,0 \%)$ não apresentaram metástases à distância $(\mathrm{p}<0,05)$, caracterizando o estádio T3N0M0 como o mais encontrado entre os 74 pacientes. O nú-
Tabela 5. Distribuição, por cirurgias a que foram submetidos, de 74 pacientes portadores de câncer colorretal submetidos a cirurgias êntero-clorretais, por dois médicos residentes em Coloproctologia como cirurgiões principais, no Programa de Pós-Graduação "lato sensu"do Grupo de Coloproctologia da SCBH e FCMMG.

\begin{tabular}{lcc}
\hline CÂNCER COLORRETAL & N & \% \\
\hline RSA-ACR (2 com ileostoma) & 27 & 36,6 \\
HCD-AIT (2 com ileostoma) & 16 & 21,7 \\
CT-AIR & 05 & 6,7 \\
RSA-DuGr ((2 com ileostoma)) & 05 & 6,7 \\
Hartmann & 05 & 6,7 \\
Laparotomia e colostomia (1 em & 05 & 6,7 \\
alça) & & \\
Laparotomia e ileostomia & 02 & 2,7 \\
HCE-ATR & 02 & 2,7 \\
PCT-ID & 02 & 2,7 \\
AAP-CD & 02 & 2,7 \\
Pan-histerec + enterect + & 02 & 2,7 \\
ressecção de metástases & & \\
Enterectomia & 01 & 1,4 \\
Total & 74 & 100 \\
\hline
\end{tabular}

RSA-ACR = Retossigmoidectomia com Anastomose ColoRetal; $H C D-A I T=H e m i C o l e c t o m i a$ Direita com Anastomose Ileo-Transverso; RSA-ACR-Dugr: RSA-ACR com Duplo Grampeamento; $C T$-AIR $=$ Colectomia Total com Anastomose Íleo Retal; HCE-ATR = HemiColectomia Esquerda com Anastomose Transverso-Retal; PCT-ID: Procto-Colectomia Total com Ileostomia Definitva; AAP-CD: Amputação Abdômino-Perineal com Colostomia Definitiva

mero médio de gânglios encontrados nas peças cirúrgicas, no exame anatomopatológico, foi de 10,4, com extremos em 5 gânglios e em 26 destes (Figura 7).

\section{Comorbidades e morbimortalidade}

A Tabela 6 e a Figura 8 mostram a distribuição pelo tipo de cirurgia executada com vários parâmetros, destacando-se as anastomoses, as comorbidades, as complicações cirúrgicas e a mortalidade. Foram feitas 63 anastomoses $(85,1 \%)$, das quais 38 foram mecânicas (\%) e 25, manuais (\%). Houve 14 comorbidades $(18,9 \%)$ : caquexia (oito), hipertensão arterial sistêmica e diabete (duas), caquexia e abdome agudo obstrutivo (uma), caquexia com abdome agudo perfurativo (uma), obesidade (uma) e hipertensão arterial sistêmica (uma). Houve nove 
Tabela 6. Distribuição, por cirurgias a que foram submetidos, de 74 pacientes portadores de câncer colorretal submetidos a cirurgias êntero-clorretais, no tocante à gradação dos tumores (TNM), por dois médicos residentes em Coloproctologia como cirurgiões principais, no Programa de Pós-Graduação "lato sensu"do Grupo de Coloproctologia da SCBH e FCMMG.

\begin{tabular}{lcccccccccccccccc}
\hline CÂNCER & N & \% & T0 & T1 & T2 & T3 & T4 & N0 & N1 & Nx & M0 & M1 & Mx & NN0 & NN1 & NNx \\
COLORRETAL & & & & & & & & & & & & & & \\
\hline RSA-ACR (2 com Il) & 27 & 36,6 & 0 & 1 & 5 & 20 & 1 & 23 & 4 & 0 & 26 & 1 & 0 & 13 & 2 & 0 \\
HCD-AIT (2 com Il) & 16 & 21,7 & 0 & 0 & 4 & 11 & 1 & 11 & 5 & 0 & 14 & 2 & 0 & 14 & 2 & 0 \\
CT-AIR & 05 & 6,7 & 0 & 0 & 0 & 5 & 0 & 4 & 1 & 0 & 5 & 0 & 0 & 17 & 1 & 0 \\
RSA-DuGr (2 com I1) & 05 & 6,7 & 0 & 0 & 1 & 3 & 1 & 2 & 3 & 0 & 5 & 0 & 0 & 14 & 2 & 0 \\
Hartamann & 05 & 6,7 & 0 & 0 & 0 & 4 & 1 & 1 & 4 & 0 & 2 & 3 & 0 & 11 & 1 & 0 \\
Lap e colostomia (1 & 05 & 6,7 & 0 & 0 & 0 & 0 & 5 & 0 & 1 & 4 & 0 & 4 & 1 & & & 5 \\
em alça) & & & & & & & & & & & & & & & & \\
Lap e ileostoia & 02 & 2,7 & 0 & 0 & 0 & 0 & 2 & 0 & 1 & 1 & 0 & 1 & 1 & & & 2 \\
HCE-ATR & 02 & 2,7 & 0 & 0 & 1 & 1 & 0 & 1 & 1 & 0 & 2 & 0 & 0 & 14 & 1 & 0 \\
PCT-ID & 02 & 2,7 & 0 & 0 & 1 & 1 & 0 & 1 & 1 & 0 & 1 & 1 & 0 & 18 & 3 & 0 \\
AAP-CD & 02 & 2,7 & 0 & 0 & 1 & 1 & 0 & 1 & 1 & 0 & 2 & 0 & 0 & 12 & & 0 \\
Pan-hist+enter+ res & 02 & 2,7 & 0 & 0 & 0 & 0 & 2 & 0 & 1 & 1 & 0 & 1 & 1 & 13 & 2 & 0 \\
metástase & & & & & & & & & & & & & & & & \\
Enterectomia & 01 & 1,4 & 1 & 0 & 0 & 0 & 0 & 0 & 0 & 1 & 0 & 0 & 1 & & & \\
Total & 74 & 100 & 1 & 1 & 13 & 46 & 13 & 44 & 23 & 7 & 57 & 13 & 4 & 13,8 & 1,9 & 7 \\
\hline
\end{tabular}

TNM em N-74: T3(46;62,1\%)=p<0,05; NO(44;59,5\%) $=p<0,05 ;$ MO $(57 ; 77,0 \%)=p<0,05 ;$ T3n0M0

Siglas usadas

RSA-ACR $=$ RetoSigmoidectomia Abdominal com Anastomose ColoRetal;

HCD-AIT = HemiColectomia Direita com Anastomose Ileo-Transverso;

RSA-ACR-DuGr: RSA-ACR com Duplo Grampeamento;

$C T$-AIR = Colectomia Total com Anastomose Ileo-Retal;

HCE-ATR = HemiColectomia Esuqerda com Anastomose Transverso-Retal.

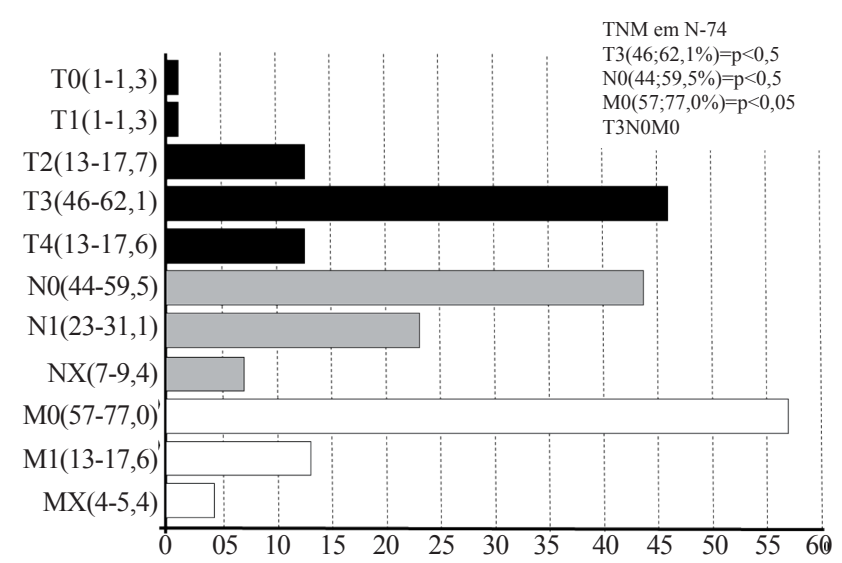

Figura 6. Distribuição, por cirurgias a que foram submetidos, de 74 pacientes portadores de câncer colorretal, submetidos a cirurgias êntero-colaterais, no tocante à gradação dos tumors (TNM), por dois médicos residentesem Coloproctologia como cirurgiões principais, no Programa de Pós Graduação "lato sensus" do Grupo de Colocproctologia da SCBH e FCMMG.

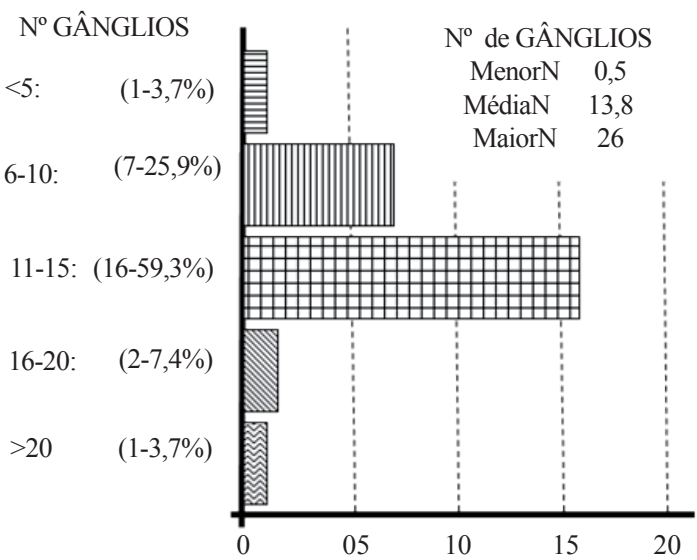

Figura 7. Distribuição, por quantidade de gânglios encontrados nas peças cirúrgicas, de 74 pacientes portadores de câncer colorretal, submetidos a cirurgias êntero-colorretais, por dois médicos residentes em Coloproctologia como cirurgiões principais, no Programa de Pós-Graduação "lato sensu”do Grupo de Coloproctologia da SCBH e FCMMG. 


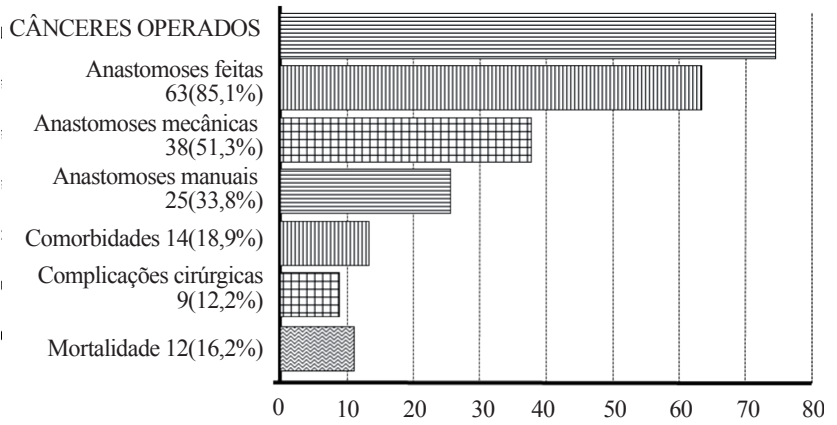

Figura 8. Distribuição, por cânceres operados (74), anastomoses feitas (63), anastomoses mecânicas (38), anastomoses manuais (25), co-morbidades dos pacientes (14), complicações cirúrgicas (9) e óbitos (12) de 74 pacientes portadores de câncer colorretal, submetidos a cirurgias êntero-colorretais, por dois médicos residentes em Coloproctologia como cirurgiões principais, no Programa de Pós-Graduação "lato sensu" do Grupo de Coloproctologia da SCBH e FCMMG.

complicações cirúrgicas (12,2\%), sendo três em 27 retossigmoidectomias com anastomose colorretal $(11,1 \%), 2$ em 16 hemicolectomia direita com anastomose íleo-transverso $(12,5 \%), 2$ em 5 colectomias totais com anastomose íleo-retal (40,0\%) e 1 em 5 retossigmoidectomias abdominais com duplo grampeamento (20\%) (Tabela 6 e Figuras 8 e 9). As complicações foram as seguintes: fístulas anastomóticas (cinco), abscessos perianastomóticos (duas), trombose mesentérica (uma) e evisceração (uma). Das nove complicações, sete ocorreram em pacientes com comorbidades e duas em pacientes sem outra doença em curso.

\section{Relação entre cirurgia realizada e algumas características do ato cirúrgico e da peça cirúrgica}

A Tabela 7 caracteriza os vários tipos de cirurgias a que os pacientes foram submetidos, relacionando-os com o tempo cirúrgico, a permanência no CTI e hospitalar, os dias de liberação da dieta, as dimensões das peças cirúrgicas e a extensão da laparotomia (Tabela 8 e Figura 11). As cirurgias que demandaram menos tempo foram as laparotomia com ileostomia (média de 75 minutos) e as com colostomia (média de 95 minutos), sendo os maiores tempos ocupados pela proctocolectomia total com ileostomia definitiva (240 minutos) e as hemicolectomias esquerdas, com anastomose transverso-retal (240 minutos), sendo o tempo médio de todas elas 160 minutos (Tabela 6 e Figura 10). Os menores

\section{CIRURGIAS REALIZADAS E COMPLICAÇÕES}

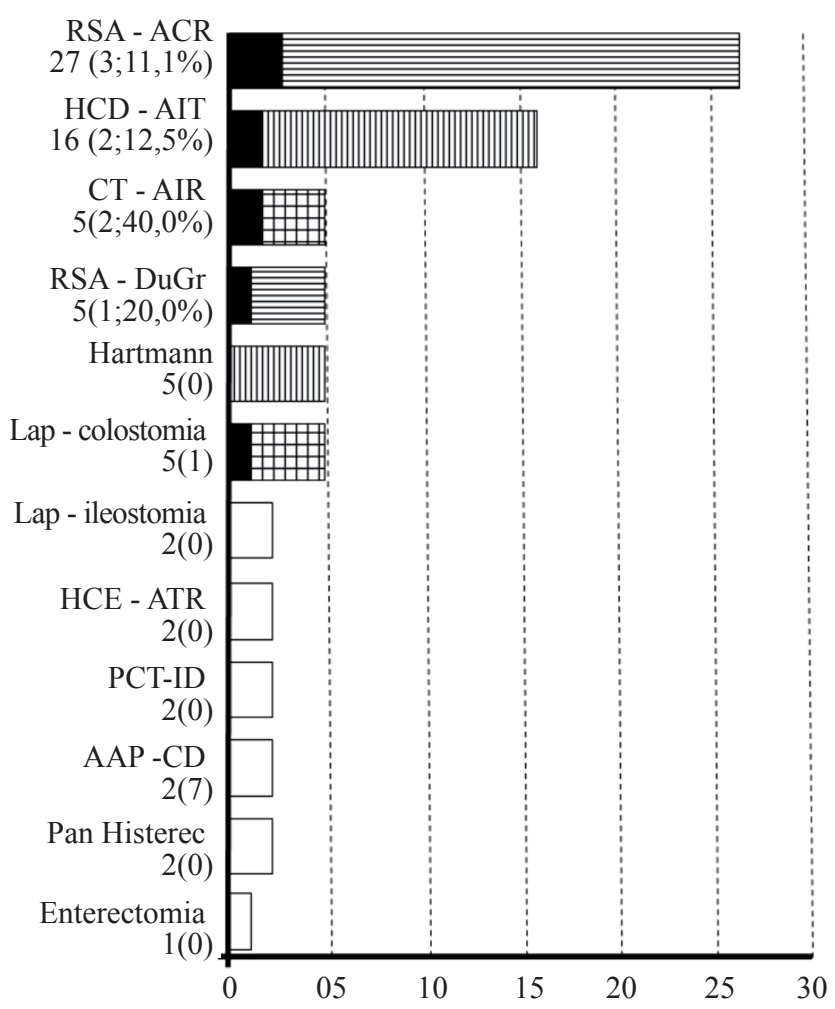

RSA-ACR: retosigmoidectomia abdominal com anastomose coloretal; HCD-AIT: hemicolectomia direita com anastomose íleotransverso; RSA-ACR-DuGr: RSA-ACR com duplo grampeamento; CT-AIR: colectomia total comanastomose ileo-retal; HCE-ATR: hemicolectomia esquerda com anastomose transverso-retal; $P C T$ ID: proctocolectomia total com ileostomia definitiva; $A A P-C D$ : amputação abdominoperineal com colostomia definitiva.

Figura 9. Distribuição, por complicações cirúrgicas decorrentes das cirurgias a que foram submetidos, de 74 pacientes portadores de câncer colorretal, submetidos a cirurgias êntero-colorretais, por dois médicos residentes em Coloproctologia como cirurgiões principais, no Programa de Pós-Graduação lato sensu, do Grupo de Coloproctologia da Santa Casa de Belo Horizonte.

períodos de internamento ocorreram nas laparotomias com ileostomia (média de dois dias), e os mais longos com as pan-histerectomias (13,5 dias), sendo a média da permanência de 6,7 dias. Quanto às extensões das peças cirúrgicas, as menores, excluindo-se uma enterectomia de $15 \mathrm{~cm}$, foram as peças de Hartmann (29 $\mathrm{cm})$, de retossigmoidectomia abdominal $(32 \mathrm{~cm})$ e as mais extensas as peças de colectomia total com anastomose íleo-retal $(120 \mathrm{~cm})$ e proctocolectomia total com 
Tabela 7. Distribuição, por cirurgias a que foram submetidos, de 74 pacientes portadores de câncer colorretal submetidos a cirurgias êntero-clorretais, no tocante às anastomoses (manual e mecânica), complicações cirúrgicas, comorbidades e óbitos, por dois médicos residentes em Coloproctologia como cirurgiões principais, no Programa de Pós-Graduação "lato sensu”do Grupo de Coloproctologia da SCBH e FCMMG.

\begin{tabular}{|c|c|c|c|c|c|c|c|c|}
\hline $\begin{array}{l}\text { CÂNCER } \\
\text { COLORRETAL } \\
\end{array}$ & $\mathbf{N}$ & $\%$ & Anastomoses & $\begin{array}{c}\text { Anast. } \\
\text { manual }\end{array}$ & $\begin{array}{l}\text { Anast } \\
\text { mec. }\end{array}$ & $\begin{array}{l}\text { Complic. } \\
\text { cirúrgica }\end{array}$ & Comorb & Óbitos \\
\hline RSA-ACR (2 com Il)* & 27 & 36,6 & 27 & 3 & 24 & 3 & 2 & 1 \\
\hline HCD-AIT (2 com Il)** & 16 & 21,7 & 16 & 11 & 5 & 2 & 1 & 2 \\
\hline CT-AIR*** & 05 & 6,7 & 05 & 1 & 4 & 2 & 1 & 0 \\
\hline RSA-DuGr $(2 \text { com Il })^{\S}$ & 05 & 6,7 & 05 & 0 & 0 & 1 & 1 & 0 \\
\hline Hartamann $n^{\S}$ & 05 & 6,7 & 05 & 5 & 0 & 0 & 2 & 2 \\
\hline $\begin{array}{l}\text { Lap e colostomia (1 } \\
\text { em alça) }\end{array}$ & 05 & 6,7 & 00 & 0 & 0 & 1 & 4 & 4 \\
\hline Lap e ileostoia ${ }^{\#}$ & 02 & 2,7 & 00 & 0 & 0 & 0 & 1 & 1 \\
\hline HCE-ATR & 02 & 2,7 & 02 & 2 & 0 & 0 & 0 & 0 \\
\hline PCT-ID $\#$ & 02 & 2,7 & 00 & 0 & 0 & 0 & 1 & 1 \\
\hline AAP-CD & 02 & 2,7 & 00 & 0 & 0 & 0 & 1 & 0 \\
\hline $\begin{array}{l}\text { Pan-hist+enter+ res } \\
\text { metástase }\end{array}$ & 02 & 2,7 & 02 & 2 & 0 & 0 & 1 & 1 \\
\hline Enterectomia $^{+}$ & 01 & 1,4 & 01 & 1 & 0 & 0 & 0 & 0 \\
\hline Total & 74 & 100 & 63 & 25 & 38 & 9 & 14 & 12 \\
\hline
\end{tabular}

Siglas usadas: RSA-ACR = RetoSigmoidectomia Abdominal com Anastomose ColoRetal; HCD-AIT = HemiColectomia Direita com Anastomose Ileo-Transverso; RSA-ACR-DuGr: RSA-ACR com Duplo Grampeamento; CT-AIR = Colectomia Total com Anastomose IleoRetal; HCE-ATR = HemiColectomia Esuqerda com Anastomose Transverso-Retal; PCT-ID: Procto-Colectomia Total com Ileostomia Definitiva; APP-CD: Amputação Abdômino-Perineal com Colostomia Definitiva

ileostomia definitiva $(150 \mathrm{~cm})$, ficando a média em 34,5 $\mathrm{cm}$ (Tabela 7, Figuras 12 e 13). Quanto às extensões das incisões abdominais, houve muita semelhança independente da técnica cirúrgica usada, variando de 25 a 35 $\mathrm{cm}$, com média de $30,5 \mathrm{~cm}$.

\section{Mortalidade}

Houve 12 óbitos (16,2\%), dois dos quais diretamente relacionados à cirurgia (um caso de deiscência de anastomose e um de evisceração); três relacionados a complicações de ordem clínica (dois casos de tromboembolismo pulmonar - TEP e um de broncopneumonia); e sete a comorbidades, cinco das quais crônicas (um caso de obesidade com hipertensão e quatro casos de caquexia e desnutrição) e duas agudas (um paciente com abdome agudo perfurativo e outro com abdome agudo obstrutivo).

\section{Sumário dos resultados}

A análise retrospectiva de 74 prontuários de pacientes do SUS, operados de câncer colorretal pelo
Residente R2, supervisionado e auxiliado por preceptores, permitiu as seguintes conclusões:

- A média etária dos pacientes foi 57,2 , sendo os tumores mais comuns em mulheres $(54,1 \%)$, localizando-se, sobretudo, no sigmoide $(31,1 \%)$, no reto alto $(24,3 \%)$ e no ceco $(17,6 \%)$.

- $\quad$ A incidência do câncer colorretal entre os 129 pacientes operados por várias doenças colorretais foi $57,4 \%$ ( 74 casos).

- As cirurgias mais realizadas foram a retossigmoidectomia com anastomose colorretal $(36,6 \%)$ e hemicolectomia direita com anastomose íleotransverso $(21,7 \%)$.

- As características anatômicas (TNM) mais comuns dos tumores foram T3 (62,1\%), N0 (59,5\%) e M0 $(77,0 \%)(p<0,05)$, sendo o número médio de gânglios encontrados nas peças cirúrgicas equivalente a 10,4 .

- $\quad$ Foram feitas 63 anastomoses $(85,1 \%)$, das quais $38(60,3 \%)$ foram mecânicas e 25 , manuais $(39,7 \%)$. 


\section{CIRURGIAS}

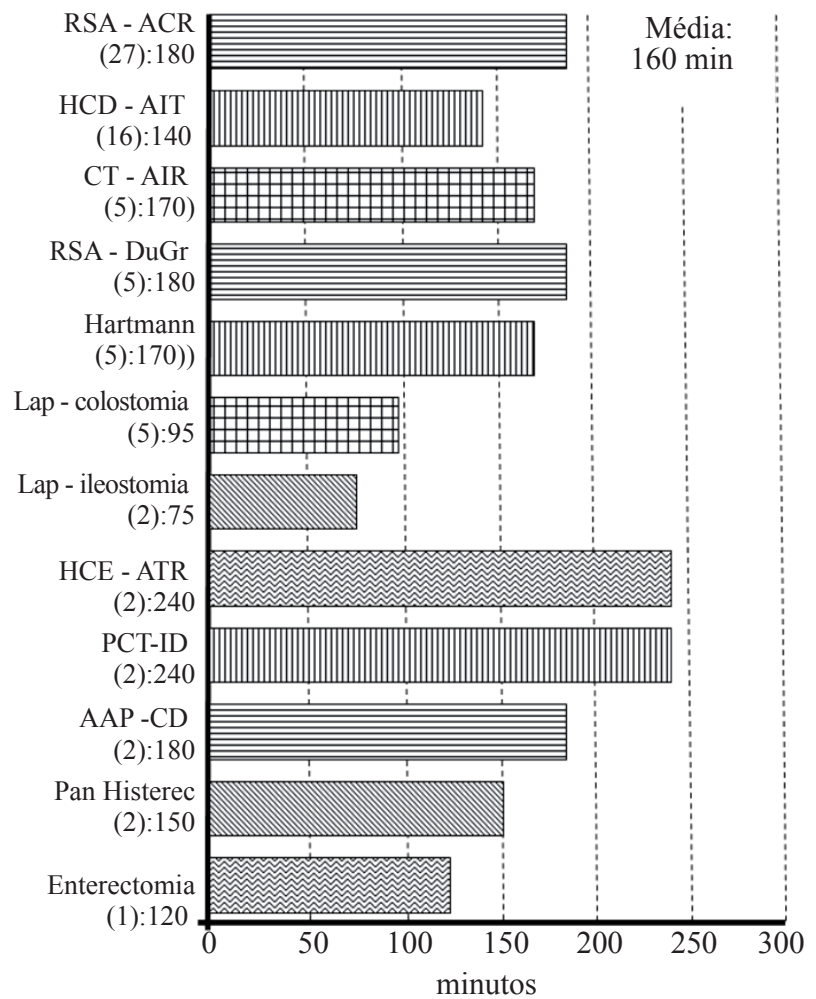

RSA-ACR: retosigmoidectomia abdominal com anastomose coloretal; HCD-AIT: hemicolectomia direita com anastomose ileotransverso; RSA-ACR-DuGr: RSA-ACR com duplo grampeamento; CT-AIR: colectomia total comanastomose íleo-retal; HCE-ATR: hemicolectomia esquerda com anastomose transverso-retal; $P C T$ ID: proctocolectomia total com ileostomia definitiva; $A A P-C D$ : amputação abdominoperineal com colostomia definitiva.

Figura 10. Distribuição, por tempo de cirurgia (em minutos), de 74 pacientes portadores de câncer colorretal, submetidos a cirurgias êntero-colorretais, por dois médicos residentes em Coloproctologia como cirurgiões principais, no Programa de Pós-Graduação lato sensu, do Grupo de Coloproctologia da Santa Casa de Belo Horizonte.

- $\quad$ A incidência das comorbidades foi de $18,9 \%$.

- A incidência de complicações cirúrgicas foi de $12,2 \%$ (nove casos), e decorreram mais das comorbidades (sete) que do ato cirúrgico (duas).

- As cirurgias menos demoradas foram as laparotomias com ileostomia (média de 75 minutos) e as com colostomia (média de 95 minutos); e as mais demoradas foram a proctocolectomia total com ileostomia definitiva (240 minutos) e as hemicolectomias esquerdas com anastomose transverso-retal (240 minutos), sendo o tempo médio delas 160 minutos.

- As menores peças cirúrgicas foram as decorrentes da cirurgia de Hartmann $(29 \mathrm{~cm})$; e as mais extensas, as peças de proctocolectomia total com ileostomia definitiva $(150 \mathrm{~cm})$, ficando a média em 34,5.

- Houve 12 óbitos (16,2\%), dois dos quais diretamente relacionados à cirurgia.

\section{DISCUSSÃO}

O câncer colorretal é o terceiro câncer mais diagnosticado, tanto em homens, quanto em mulheres. Com relação à mortalidade, o câncer colorretal fica também com a terceira colocação entre as causas mais comuns de morte, por câncer, nos Estados Unidos ${ }^{1}$. Estatísticas americanas mostram que 26.801 homens e 26.395 mulheres faleceram por câncer colorretal nos Estados Unidos, em $2006^{2}$.

Na presente casuística, a média de idade e os picos de incidência do diagnóstico foram comparáveis àqueles descritos na literatura ${ }^{3}$. Com relação à localização dos tumores, a maioria $(66,2 \%)$ se localizava no reto e sigmoide. $\mathrm{O}$ exame proctológico completo realizado no Brasil, que inclui a realização de retossigmoidoscopia rígida até $25 \mathrm{~cm}$ acima do ânus, pode ser útil na identificação de lesões nessas localizações. Tumores proximais têm sido responsáveis por uma parcela maior dos casos na literatura. Rim et al. (2009) encontraram tumores de cólon proximais, responsáveis por $42,3 \%$ dos casos de câncer colorretal, ficando à frente dos tumores do cólon distal $(25,3 \%)$ e do reto $(27,4 \%)^{4}$.

Dados sugerem que o emprego dos métodos de rastreamento para o câncer colorretal tem impacto positivo na redução da incidência e mortalidade ${ }^{5}$. Nos Estados Unidos, houve redução na incidência e mortalidade por câncer colorretal nos últimos anos. Porém, outras partes do mundo mostraram aumento da incidência dos cânceres colorretais, especialmente nos países asiáticos e no Leste Europeu ${ }^{1}$. Países desenvolvidos são os que geralmente mostram estabilização ou declínio na incidência de câncer, pois é onde ge- 


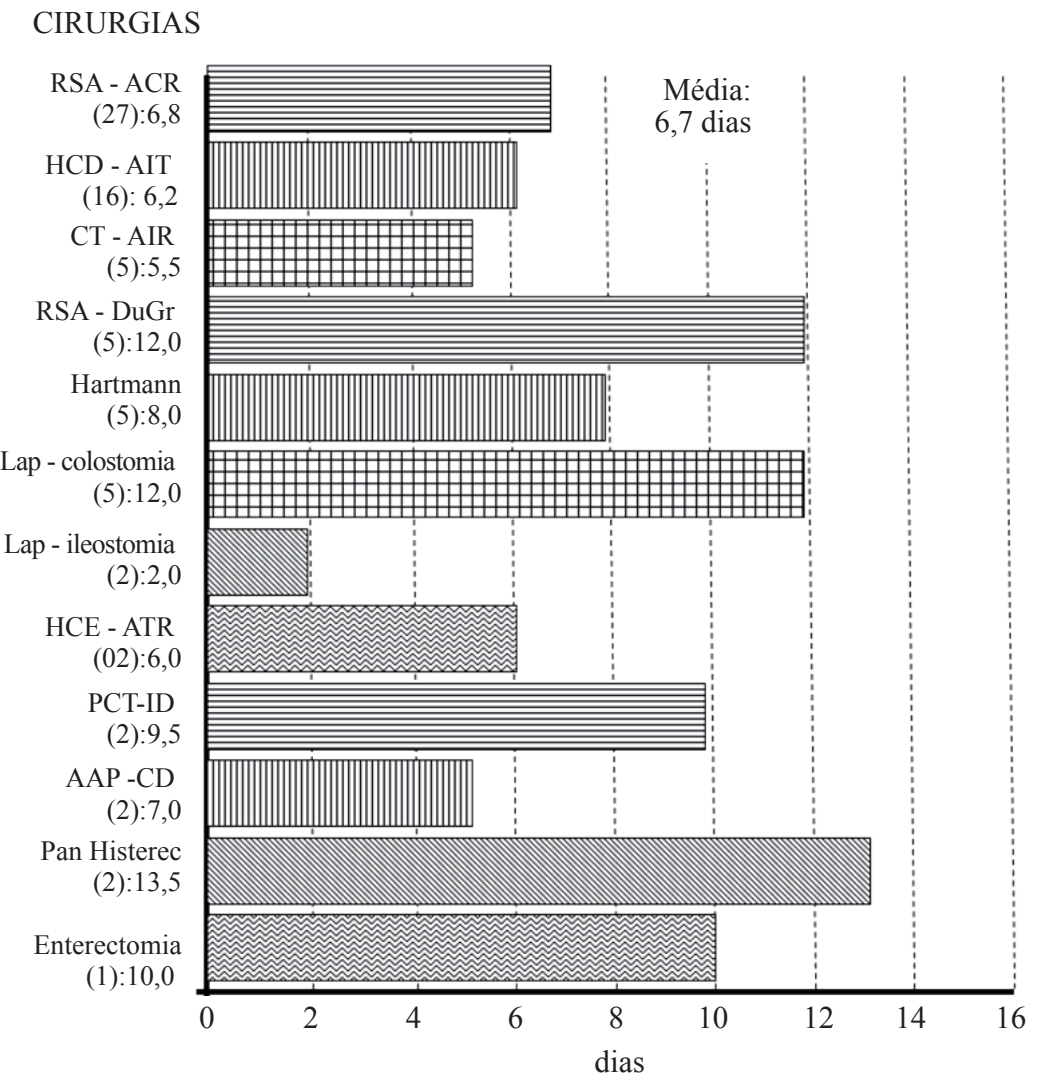

RSA-ACR: retosigmoidectomia abdominal com anastomose coloretal; HCD-AIT: hemicolectomia direita com anastomose ileo-transverso; RSA-ACR-DuGr: RSA-ACR com duplo grampeamento; CT-AIR: colectomia total comanastomose ileo-retal; HCE-ATR: hemicolectomia esquerda com anastomose transverso-retal; PCT-ID: proctocolectomia total com ileostomia definitiva; AAP-CD: amputação abdominoperineal com colostomia definitiva.

Figura 11. Distribuição, por tempo de internamento hospitalar (em dias), de 74 pacientes portadores de câncer colorretal, submetidos a cirurgias êntero-colorretais, por dois médicos residentes em Coloproctologia como cirurgiões principais, no Programa de Pós-Graduação lato sensu, do Grupo de Coloproctologia da Santa Casa de Belo Horizonte.

ralmente são adotados programas de rastreamento da população, como na Europa, nos Estados Unidos, em Israel e na Austrália.

No presente estudo, os pacientes estudados foram atendidos pelo SUS. Mesmo com a existência dos exames de rastreamento disponíveis, nem todos conseguem a realização de exames mais complexos como colonoscopia ou consulta com especialista, devido à demanda reprimida. Este atraso ou mesmo a eventual falta de acesso aos exames podem ser responsáveis pelo diagnóstico da maioria dos tumores já com estadiamento T avançado. A soma dos tumores T3 e T4 totalizou $79,7 \%$ dos casos. Em outras partes do mundo, também verificou-se pouco acesso aos programas de rastreamento por populações menos favorecidas, como confirma relatório americano de $2006^{6}$.

A maioria dos pacientes (59,5\%) não apresentava sinais de metástases para linfonodos, porém o número médio de linfonodos examinados foi de 10,4. Na literatura, é descrita a melhora do prognóstico de tumores T3 quando se consegue avaliação de maior número de gânglios na peça cirúrgica. Swanson et al. (2003) sugerem que o número mínimo de linfonodos avaliados seja de 13, antes de se classificar um tumor T3 como $\mathrm{N} 0^{7}$. No presente estudo, o número médio de linfonodos examinados foi inferior ao recomendado. Porém, ainda não há tempo decorrido o suficiente para avaliação de sobrevida após longo prazo. 
Cirurgia no câncer colorretal-abordagem cirúrgica de 74 pacientes do SUS portadores de câncer colorretal em programa de pós-graduação lato sensu em coloproctologia Rodrigo guimarães oliveira e Cols.
Vol. 31 $\mathbf{N}^{0} 1$

\section{CIRURGIAS}

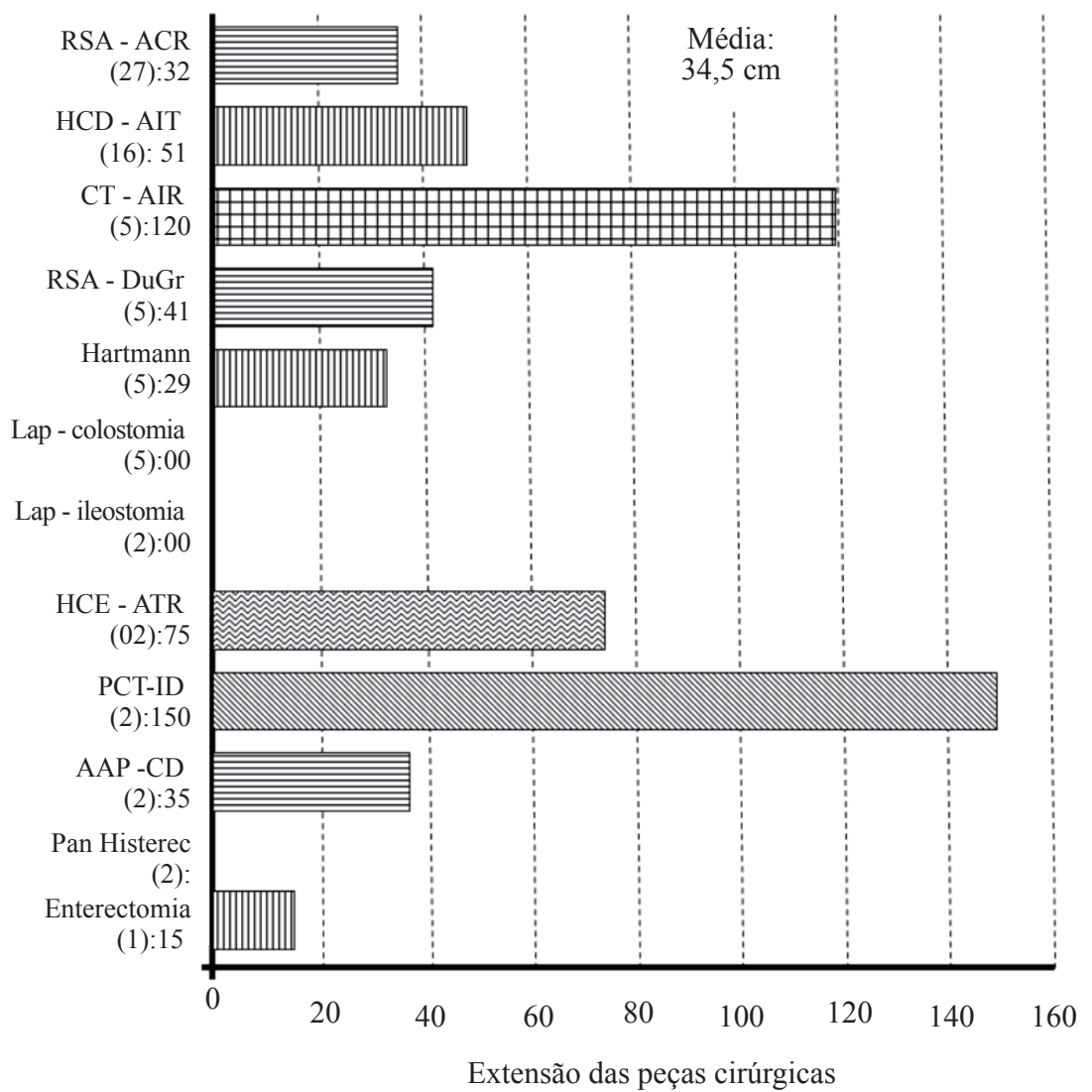

RSA-ACR: retosigmoidectomia abdominal com anastomose colorretal; HCD-AIT: hemicolectomia direita com anastomose íleotransverso; RSA-ACR-DuGr: RSA-ACR com duplo grampeamento; CT-AIR: colectomia total comanastomose ileo-retal; HCE-ATR: hemicolectomia esquerda com anastomose transverso-retal; PCT-ID: proctocolectomia total com ileostomia definitiva; AAP-CD: amputação abdominoperineal com colostomia definitiva.

Figura 12. Distribuição, por extensão das peças cirúrgicas (em centímetros), de 74 pacientes portadores de câncer colorretal, submetidos a cirurgias êntero-colorretais, por dois médicos residentes em Coloproctologia como cirurgiões principais, no Programa de Pós-Graduação lato sensu, do Grupo de Coloproctologia da Santa Casa de Belo Horizonte.

A presença de comorbidades associadas à doença de base pode ser esperada na faixa etária mais acometida pelos tumores colorretais. Dentre as complicações decorrentes das operações colorretais, a deiscência e fístula anastomóticas costumam ser consideradas as mais graves ${ }^{8-10}$. A incidência das fístulas anastomóticas em 63 cirurgias colorretais envolvendo anastomoses foi de cinco casos (7,9\%). A incidência deste tipo de complicação varia muito na literatura, sendo descritas desde 1 a 39\%. Porém, com percentual menor, tendo significado clínico. Estudos mostram incidência de fístulas em torno de $6 \%$ após os procedimentos colônicos e $19 \%$, após procedimentos retais $^{11,12}$. Komen et al.(2009) encontraram 8,7\% de fístulas em 739 anastomoses colorretais, identificando o tempo cirúrgico prolongado e o índice de massa corporal (IMC) maior que $30 \mathrm{~kg} / \mathrm{m}^{2}$ como fatores de risco independentes para a ocorrência de fístulas ${ }^{10}$. Outros fatores de risco identificados são: presença de doença inflamatória intestinal, operações retais, risco cirúrgico aumentado (classificação da ASA), hipoalbuminemia, perda sanguínea intraoperatória aumentada e hemotransfusão ${ }^{8,9}$.

Quanto à mortalidade após operações colorretais, houve duas mortes relacionadas diretamente ao procedimento operatório e dez mortes relacionadas à 
Cirurgia no câncer colorretal - abordagem cirúrgica de 74 pacientes do SUS portadores de câncer colorretal em programa de pós-graduação lato sensu em coloproctologia Rodrigo guimarães oliveira e Cols.
Vol. 31 $\mathbf{N}^{\circ} 1$

CIRURGIAS

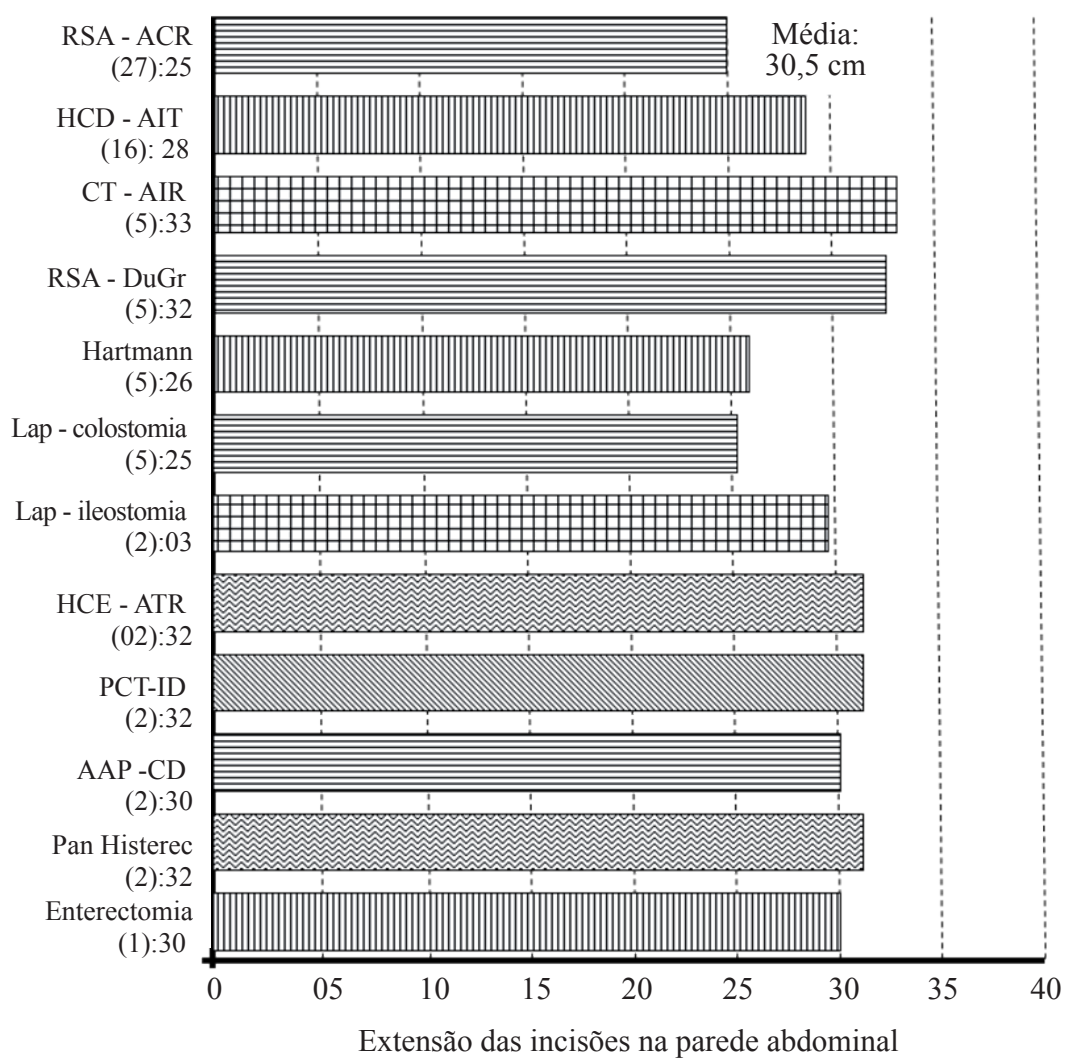

RSA-ACR: retosigmoidectomia abdominal com anastomose coloretal; HCD-AIT: hemicolectomia direita com anastomose ileo-transverso; RSA-ACR-DuGr: RSA-ACR com duplo grampeamento; CT-AIR: colectomia total comanastomose íleo-retal; HCE-ATR: hemicolectomia esquerda com anastomose transverso-retal; PCT-ID: proctocolectomia total com ileostomia definitiva; AAP-CD: amputação abdominoperineal com colostomia definitiva.

Figura 13. Distribuição, por extensão das incisões cutâneas (em centímetros), de 74 pacientes portadores de câncer colorretal, submetidos a cirurgias êntero-colorretais, por dois médicos residentes em Coloproctologia como cirurgiões principais, no Programa de Pós-Graduação "sensu lato" do Grupo de Coloproctologia da Santa Casa de Belo Horizonte.

presença de comorbidades apresentadas pelos pacientes. Taxas de mortalidade de pacientes no pós-operatório de cirurgia colorretal por câncer costumam ser estimadas em cerca de até $3 \%{ }^{13,14}$. Fatores que poderiam influenciar nesta mortalidade aumentada seriam a inclusão de casos operados em caráter de urgência, as condições de saúde da população atendida, a presença de cirurgiões em formação e a própria estrutura da instituição. Uma análise mais adequada dos resultados ainda poderá ser obtida com o acompanhamento dos pacientes incluídos no presente estudo, após obtenção dos dados de seguimento mais longo, como, por exemplo, a sobrevida em cinco anos.
A análise retrospectiva de 74 prontuários dos pacientes do SUS, operados de câncer colorretal pelo Residente R2 supervisionado e auxiliado por preceptores, permitiu verificar que os tumores foram mais comuns entre mulheres e na sexta década de vida, as cirurgias mais realizadas foram as retossigmoidectomias abdominais com anastomose colorretal; as características anatomopatológicas dos tumores foram tumores invasores de musculatura própria sem gânglios comprometidos e sem metástases; e a elevada incidência de complicações cirúrgicas decorreu, principalmente, do mau estado geral dos pacientes e das elevadas taxas de comorbidades dos portadores. 
Tabela 8. Distribuição, por cirurgias realizadas,em 74 pacientes portadores de câncer colorretal submetidos a cirurgias êntero-clorretais, no tocante ao tempo de cirurgia, dias em CTI, dias de internamento hospitalar, dia de liberação de dieta, extensão da peça e da incisão cirúrgica abdominal, realizadas por dois médicos residentes em Coloproctologia como cirurgiões principais, no Programa de Pós-Graduação "lato sensu"do Grupo de Coloproctologia da SCBH e FCMMG.

\begin{tabular}{|c|c|c|c|c|c|c|c|c|}
\hline $\begin{array}{l}\text { CÂNCER } \\
\text { COLORRETAL }\end{array}$ & $\mathbf{N}$ & $\%$ & Tempo (min) & CTI(N) & $\begin{array}{c}\text { Hospital } \\
\text { (dias) }\end{array}$ & $\begin{array}{l}\text { Dieta } \\
\text { (dias) }\end{array}$ & $\begin{array}{l}\begin{array}{l}\text { Peça } \\
(\mathrm{cm})\end{array} \\
\end{array}$ & $\begin{array}{c}\text { Incisão } \\
(\mathbf{c m})\end{array}$ \\
\hline RSA-ACR (2 com Il) & 27 & 36,6 & 180 & $18-66,7 \%$ & $6,8(14)$ & 2,1 & 32 & 25 \\
\hline HCD-AIT (2 com Il) & 16 & 21,7 & 140 & $06-37,5 \%$ & $6,2(11)$ & 2,3 & 51 & 28 \\
\hline CT-AIR & 05 & 6,7 & 170 & $02-40,0 \%$ & $5,5(10)$ & 2,1 & 120 & 33 \\
\hline RSA-DuGr (2 com Il) & 05 & 6,7 & 180 & $03-60,0 \%$ & $12(28)$ & 3,0 & 41 & 32 \\
\hline Hartamann & 05 & 6,7 & 170 & $01-20,0 \%$ & $8(14)$ & 2,1 & 29 & 26 \\
\hline $\begin{array}{l}\text { Lap e colostomia ( } 1 \\
\text { em alça) }\end{array}$ & 05 & 6,7 & 095 & $03-60,0 \%$ & $12(20)$ & & & 25 \\
\hline Lap e ileostoia & 02 & 2,7 & 075 & $01-50,0 \%$ & 2 & & & 30 \\
\hline HCE-ATR & 02 & 2,7 & 240 & $01-50,0 \%$ & 6 & 2 & 75 & 32 \\
\hline PCT-ID & 02 & 2,7 & 240 & $00-0 \%$ & 9,5 & 1,5 & 150 & 32 \\
\hline AAP-CD & 02 & 2,7 & 180 & $02-100 \%$ & 7 & 2 & 35 & 30 \\
\hline $\begin{array}{l}\text { Pan-hist+enter+ res } \\
\text { metástase }\end{array}$ & 02 & 2,7 & 150 & $0-0 \%$ & $13,5(20)$ & 1,5 & & 32 \\
\hline Enterectomia & 01 & 1,4 & 120 & $0-0 \%$ & 10 & 2 & 15 & 30 \\
\hline Total & 74 & 100 & 160 & $37-50,0 \%$ & 6,7 & 2,1 & 34,5 & 30,5 \\
\hline
\end{tabular}

Siglas usadas: RSA-ACR = RetoSigmoidectomia Abdominal com Anastomose ColoRetal; HCD-AIT = HemiColectomia Direita com Anastomose Ileo-Transverso; RSA-ACR-DuGr: RSA-ACR com Duplo Grampeamento; CT-AIR = Colectomia Total com Anastomose IleoRetal; HCE-ATR = HemiColectomia Esuqerda com Anastomose Transverso-Retal; PCT-ID: Procto-Colectomia Total com Ileostomia Definitiva; APP-CD: Amputação Abdômino-Perineal com Colostomia Definitiva

\section{CONCLUSÃO}

A análise retrospectiva de 74 prontuários de pacientes do SUS, operados de câncer colorretal pelo Residente R2 supervisionado e auxiliado por preceptores, permitiu afirmar que a maioria dos pacientes era do sexo feminino, na sexta década de vida, sendo o câncer colorretal prevalente entre os vários diagnósticos, tendo sido a retossigmoidectomia abdominal com anastomose colorretal a cirurgia mais realizada, com prevalência de tumores T3N0M0, decorrendo a elevada taxa de morbimortalidade ao mau estado de saúde e elevado índice de comorbidades dos pacientes.

ABSTRACT: In the framework of postgraduate Coloproctology for 2009, two graduate students conducted the second year as principal surgeons, 129 major surgeries, always assisted effectively by one or two tutors. All surgeries were performed on public patients in Santa Casa de Belo Horizonte, with absolute presence of members of the Grupo de Coloproctologia da Santa Casa de Belo Horizonte e Faculdade de Ciências Médicas de Minas Gerais (GCP-CBHS-FCMMG). A retrospective analysis of 74 medical records of patients from the Brazilian National Health System, resected of colorectal cancer by Resident R2, supervised and assisted by mentors, could get into the following conclusions: the average age of patients was 57.2, and the sixth and seventh decades accounted for $\mathbf{5 1 . 4 \%}$ of the patients. The rectal cancer was predominant in women $(\mathbf{5 4 . 1 \% )}$. The most common sites of tumors were in the sigmoid $(31.1 \%)$, rectum $(\mathbf{2 4 . 3 \%})$, and cecum $(\mathbf{1 7 . 6 \% )}$. The most commonly performed procedures were retossigmoidectomy with colorectal anastomosis $(\mathbf{3 6 . 6 \% )}$ and right hemicolectomy with ileo-transverse anastomosis $(21.7 \%)$. The anatomical characteristics of the tumors based on TNM classification findings were: T3 (62.1\%), N0 (59.5\%), and M0 (77.0\%) 
$(\mathbf{p}<\mathbf{0 . 0 5})$. The average number of lymph nodes found in surgical specimens was 10.4 . Sixty-three anastomoses $(85.1 \%)$ were carried out, being $38(60.3 \%)$ mechanical and 25 , manual (39.7\%). There were 14 comorbidities (18.9\%), the cachexia having more prominence (eight cases). The rate of surgical complications was $12.2 \%$ (nine cases), and the surgeries that have caused more complications were total colectomy with ileo-rectal anastomosis $\mathbf{( 4 0 . 0 \% )}$, and double stapled abdominal retossigmoidectomy $\mathbf{( 2 0} \%)$. The most common complications were anastomotic fistula (five cases). Complications (nine) were more caused by comorbidities (seven) than by the surgical procedure (two). The surgeries that required less time were: laparotomy with ileostomy (average of 75 minutes) and with colostomy (average of 95 minutes), and the longest times were occupied by total proctocolectomy with definite ileostomy (240 minutes) and left hemicolectomy with transverse-rectal anastomosis (240 minutes), with an average length of 160 minutes. The smaller specimens were those resulting from Hartmann's procedure (29 $\mathrm{cm})$ and retossigmoidectomy $(32 \mathrm{~cm})$; and the most extensive specimens were of total colectomy with ileorectal anastomosis $(120 \mathrm{~cm})$ and total proctocolectomy with terminal ileostomy $(150 \mathrm{~cm})$, with the mean at 34.5 . There were 12 deaths $(16.2 \%)$, two of which directly related to surgery and the other related to clinical complications and comorbities.

Keywords: colorectal cancer; colorectal surgery; cancer; Unified Health System.

\section{REFERÊNCIAS}

1. Gellad ZF, Provenzale D. Colorectal Cancer: National and International Perspective on the Burden of Disease and Public Health Impact. Gastroenterology 2010;138:2177-90.

2. Colorectal Cancer Statistics. Disponível em: <http://www. cdc.gov/cancer/ colorectal/statistics/index.htm. Acesso em: 20 mai 2010.

3. Xu AG, Yu ZJ, Jiang B, Wang XY, Zhong XH, Liu JH, et al. Colorectal cancer in Guangdong Province of China: A demographic and anatomic survey. World J Gastroenterol 2010;16:960-5.

4. Rim SH, Seeff L, Ahmed F, King JB, Coughlin SS. Colorectal cancer incidence in the United States, 1999-2004 : an updated analysis of data from the National Program of Cancer Registries and the Surveillance, Epidemiology, and End Results Program. Cancer 2009;115:1967-76.

5. Bresalier RS. Early detection of and screening for colorectal neoplasia. Gut Liver 2009;3:69-80.

6. Use of colorectal cancer tests - United States, 2002, 2004, and 2006. MMWR Morb Mortal Wkly Rep 2008;57:253-8.

7. Swanson RS, Compton CC, Stewart AK, Bland KL. The prognosis of T3N0 colon cancer is dependent on the number of lymph nodes examined. Ann Surg Oncol 2003;10:65-71.

8. Telem DA, Chin EH, Nguyen SQ, Divino CM. Risk Factors for Anastomotic Leak Following Colorectal Surgery: A CaseControl Study. Arch Surg 2010;145:371-6.

9. Buchs NC, Gervaz P, Secic M, Bucher P, Mugnier-Konrad $\mathrm{B}$, Morel P. Incidence, consequences, and risk factors for anastomotic dehiscence after colorectal surgery: a prospective monocentric study. Int J Colorectal Dis 2008;23:265-70.

10. Komen N, Dijk JW, Lalmahomed Z, Klop K, Hop W, Kleinrensink GJ, et al. After-hours colorectal surgery: a risk factor for anastomotic leakage. Int J Colorectal Dis 2009;24:789-95.

11. Alves A, Panis Y, Trancart D, Regimbeau JM, Pocard M, Valleur P. Factors Associated with Clinically Significant Anastomotic Leakage after Large Bowel Resection: Multivariate Analysis of 707 Patients. World J Surg 2002;26:499-502.

12. Matthiessen P, Hallbook O, Rutegard J, Simert G, Sjodahl R. Defunctioning Stoma Reduces Symptomatic Anastomotic Leakage After Low Anterior Resection of the Rectum for Cancer: A Randomized Multicenter Trial. Ann Surg 2007;246:207-14.

13. Dimick JB, Cowan JA, Upchurch GR, Colletti LM. Hospital Volume and Surgical Outcomes for Elderly Patients With Colorectal Cancer in the United States. J Surg Res 2003;114:50-6.

14. Ferenchild FTJ, Dawson I, de Wwilt JHW, de Graaf EJR, Groenendijk RPR, Tetteroo GWM. Total mesorectal excision for rectal cancer in an unselected population: quality assessment in a low volume center. Int $\mathrm{J}$ Colorectal Dis 2009;24:923-9.

Endereço para correspondência: GERALDO MAGELA GOMES DA CRUZ Rua Rio de Janeiro, 2.017, 1p. 1401 - Lourdes CEP: 30160-042 - Belo Horizonte (MG), Brasil E-mail: magelacruz@terra.com.br 